\title{
Streetcar Passenger Flow Maps in Old Tokyo City in 1930
}

\author{
Kazuki Ishikawa $^{\mathrm{a}, \mathrm{b}, *}$, Daichi Nakayama ${ }^{\mathrm{a}}$ \\ ${ }^{a}$ Department of Geography, Tokyo Metropolitan University, Tokyo, Japan, ishikawa-kazuki@ed.tmu.ac.jp, daichi@tmu.ac.jp \\ ${ }^{b}$ Research Fellow of Japan Society for the Promotion of Science \\ * Corresponding author
}

Keywords: origin-destination flow, old Tokyo city, modern era, streetcar

\begin{abstract}
:
In recent years, we can easily get various data of human mobility. Based on these data, there are many studies about human mobility all over the world. In Japan, Person Trip (PT) survey has been carried out once a decade after 1960s. With the use of PT data, we can grasp people's flow, however, it is difficult to get such data in the modern era. In old Tokyo city, some traffic surveys were carried out by municipality. These data are limited, however, it is possible to figure out the people's flow in the modern era by digitizing and making maps from historical documents of these surveys. The purpose of this study is to make and analyse streetcar passenger flow maps in old Tokyo city by using origin-destination (OD) data acquired through the survey conducted in 1930.
\end{abstract}

We used the OD flow data of the streetcar passenger acquired by old Tokyo city on Tuesday, June 10th, 1930. The study area was in the centre of present Tokyo 23 wards. Although the streetcars no longer exist except for one line at present, there were a lot of routes in the city in 1930. The streetcars were the important transportation for people living inside and outside the city.

To make OD flow map, we first made digital data of the OD matrix of the passengers from historical documents of the survey. There were about 400 stops, however, some adjacent stops were aggregated to save space of the document. Therefore, the OD matrix were 289 by 289. In addition, we created GIS data of streetcar's network based on old maps and historical documents. The aggregated stops were created in a midpoint of original stops. After that, we made the OD flow map by using the visualizing method described by Wood, Dykes, \& Slingsby (2010).

Figure 1 shows OD flow map of streetcar passenger in 1930. The results of this study are summarized as follows; 1) There were many people who went to the direction of Tokyo station(E6), Nihonbashi(E7), and Ginza(F6) from the entire area of the city. These areas have been a centre of commerce since pre-modern era, being crowded with shoppers, tourists and workers. Particularly, there were many commuters around Tokyo station because it had many office buildings. 2) There were many people who used the terminal stations located outside the city. These stations such as Shinjuku(D1) and Shibuya(G1) have been nodal points of other railways in the western part of Tokyo. This result showed that the people moved to the suburb of Tokyo after the Great Kanto Earthquake in 1923. 3) The movements from the west of the city to the centre of Tokyo were longer than those from the east of the city, because the western area had the restrictive land uses such as high class residential area and military reservation which covered a vast area of the western part of the city. In contrast, the old urban area extended to the eastern part of the city since that era. 


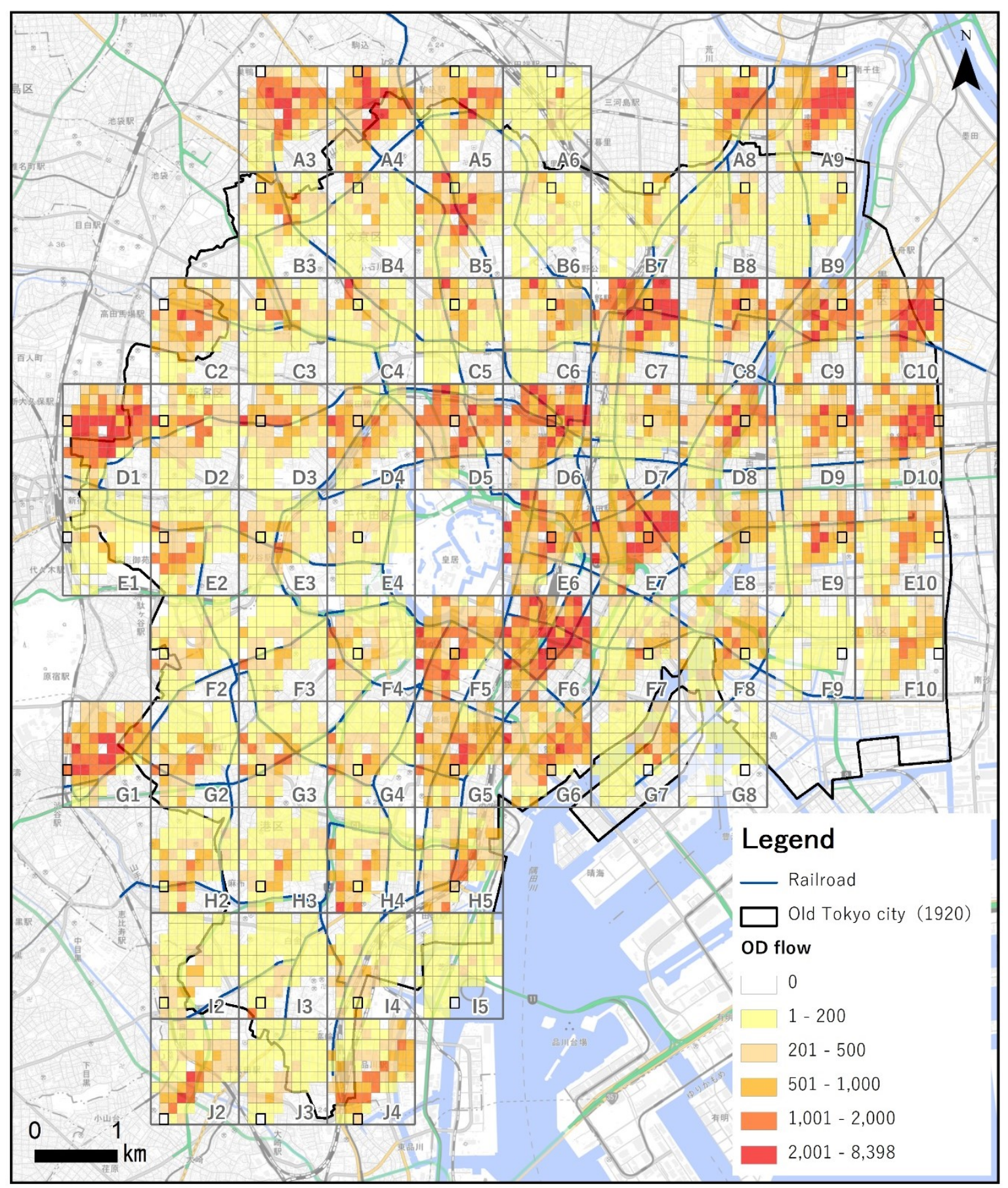

Figure 1 OD flow map of streetcar passenger in 1930 